\title{
The role of clinical geneticists in hereditary cancer management and research
}

\author{
Hanne Meijers-Heijboer* \\ Department of Clinical Genetics, Academisch Medisch Centrum - Universiteit \\ van Amsterdam (AMC-UvA) and Department of Clinical Genetics, VU University \\ Medical Center Amsterdam, The Netherlands
}

DOI: http://dx.doi.org/10.19106/JMedScieSup0048042016012

\section{ABSTRACT}

Hereditary cancer refers to cancers caused by germline mutations in cancerpredisposing genes. These mutations confer a significantly increased risk of cancer are rare and in the majority of cases, autosomal-dominantly inherited. Since the eighties of last century more than 115 cancer-predisposing genes have been identified. In many Western countries genetic testing of patients and families with clustering of cancers started early, and was often performed by clinical geneticists (MDs performed the counselling and pedigree analyses) and by molecular biologists (in laboratories within departments of clinical genetics). It turned out to be a long path to fully realize the promise of cancer-predisposing genes. The clinical utility of many cancer genetics tests and subsequent risk to reduce interventions has not yet been validated and has several pitfalls, e.g. misinterpretation of genetic variants found. However, without doubt genetic testing for mutations will eventually turn out as a important tool to save lives from early cancer-related death and become part of standard cancer care throughout the developed world. Apart from primary surgical prevention, major progress is to be expected in earlier diagnoses, tailored therapies, and possibly chemoprevention. Ideally, researchers, clinical geneticists, molecular biologists, surgeons, oncologists, gynaecologists, and other professionals will work together to reach this goal.

Keywords: clinical genetics, germline, hereditary cancers, cancer predisposing genes 